\title{
Design and analysis of a microgyroscope with sol-gel piezoelectric plate
}

\section{Guohong He $\dagger$, Christopher C T Nguyen $\dagger$, Jane C M Hui $\dagger$, S-W Ricky Lee $\ddagger \S$ and Howard C Luong $\dagger$}

$\dagger$ Department of Electrical and Electronics Engineering, Hong Kong University of Science and Technology, Clear Water Bay, Kowloon, Hong Kong

\$ Department of Mechanical Engineering, Hong Kong University of Science and Technology, Clear Water Bay, Kowloon, Hong Kong

Received 6 April 1998, in final form 5 February 1999

\begin{abstract}
A piezoelectric-plate vibration microgyroscope is proposed in this paper. Unlike other piezoelectric gyroscopes, it is based on a piezoelectric plate without the use of any elastic element. Its structure is very simple. The piezoelectric plate is excited in the polarization direction to produce the thickness extension vibration mode. If there is an input rotation on the vibrator, an induced voltage will be produced in the direction perpendicular to the plane composed of the polarization axis and the input angular rate axis according to the Coriolis effect. This arrangement allows for sensing of two-axis rotation on a single chip. A mathematical model for the microgyroscope is presented, and calculated results for a given module are provided. A macromode experiment has been carried out, and experimental results correspond very well to theoretical predictions.
\end{abstract}

(Some figures in this article appear in black and white in the printed version.)

\section{Introduction}

In inertial sensing systems, measurement of angular velocity is very important. Traditional gyroscopes such as mechanical gyroscopes, fiber optic gyroscopes, acoustic gyroscopes and vibration gyroscopes are too large and expensive for many applications, especially for aerospace and automotive applications. Macro-piezoelectric gyroscopes use relatively small piezoelectric elements attached to a large, non-piezoelectric resonator. Differences in expansion coefficients of these two materials will degrade the gyroscope performance when temperature changes. Moreover, their structures are complex, measurement set-up is complicated and assembly work is expensive.

A simple, macro-vibrating plate gyroscope has been proposed by Hansjoachim [1] which utilizes the thickness shear vibration of a piezoelectric plate to measure the input rotation rate. This design has solved some problems by enhancing the thermal stability, simplifying the structure and measurement set-up and lowering the cost of assembly work. However, as shown later, the output voltage signal of this structure is very small and is not suitable for miniaturization.

Microgyroscopes have many potential advantages. They could be mass produced and could have both driving and detecting circuits integrated on-chip for improved sensitivity.

$\S$ Correspondent for all communications. Currently on sabbatical leave at 1137-B San Antonio Road, Palo Alto, CA 94303, USA. Tel: (650) 919-0300 x102. Fax: (650) 919-0303. E-mail address: rickylee@epswin.com .
The whole gyroscope system could be very small and inexpensive. In the past few years, there has been a great deal of research on micromachined gyroscopes [2-5]. However, the vast majority of these microgyroscopes use electrostatic force to excite a vibrating structure and measure the rate of rotation by the Coriolis effect. Although there has been some progress in these microdevices, their performance is mediocre and does not meet the exact requirements of many applications. Furthermore, these devices require high DC driving voltages.

Replacing electrostatic excitation by the piezoelectric effect is an attractive option in a micro-vibration gyroscope. The energy density in piezoelectric material is very high. Therefore, the excitation voltage of vibration can be scaled down too. In this paper, a piezoelectric plate microgyroscope is proposed. It is based on the Hansjoachim gyroscope principle but is suitable for microfabrication. The micropiezoelectric gyroscope is made of a PZT in the form of a piezoelectric plate. Its structure is simple. The piezoelectric plate is excited in the polarization direction to produce the thickness extension vibration mode. According to the Coriolis effect, if there is an input rotation, an induced voltage will be produced in a perpendicular direction, and the output voltage will be introduced in the remaining perpendicular direction. This gyroscope can sense two axes of rotation on a single chip. Not only does it have all the advantages of the Hansjoachim design, but also has larger output voltage. This paper presents theoretical analysis of the device, and 


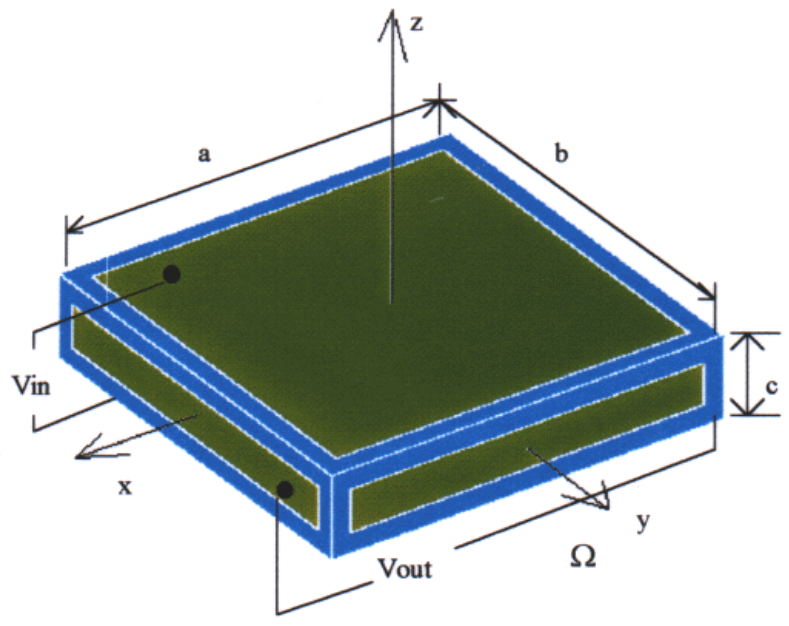

Figure 1. Piezoelectric plate gyroscope.

gives simulation results for a particular model. Experiments performed with macro-PZT models show good agreement with theoretical predictions.

\section{Piezoelectric plate microgyroscope principle}

The proposed microgyroscope uses PZT as a piezoelectric plate to sense the input rotation rate. Its arrangement is shown in figure 1. In this figure, $a, b$ and $c$ are the length, width and height of the piezoelectric plate, and they are along the $x$-axis, $y$-axis and $z$-axis, respectively. The polarization direction of the PZT is along the $z$-axis, perpendicular to the wafer plane. When an AC voltage is applied in the polarization direction, the thickness extension vibration mode is excited. If there is an angular velocity applied to this inertial system in the $y$-direction, an apparent Coriolis force will be produced in the $x-z$-plane along the $x$-axis. It is a shear stress and will cause an induced voltage in the $x$-direction whose amplitude is proportional to the input rotation rate. The Coriolis force $\vec{F}_{C}$ applied to an inertial body can be written as:

$$
\vec{F}_{C}=2 m \vec{v} \times \vec{\Omega}
$$

where $m$ is the mass of the inertial body, $\vec{v}$ is its moving velocity and $\vec{\Omega}$ is the input angular velocity.

In figure 1, with the polarization direction of the piezoelectric plate along the $z$-axis, the piezoelectric equation can be written as [6]:

$$
\left(\begin{array}{l}
S_{11} \\
S_{22} \\
S_{33} \\
S_{23} \\
S_{31} \\
S_{12} \\
D_{1} \\
D_{2} \\
D_{3}
\end{array}\right)=\left(\begin{array}{ccccccccc}
s_{11}^{E} & s_{12}^{E} & s_{13}^{E} & 0 & 0 & 0 & 0 & 0 & d_{31} \\
s_{12}^{E} & s_{11}^{E} & s_{13}^{E} & 0 & 0 & 0 & 0 & 0 & d_{31} \\
s_{13}^{E} & s_{13}^{E} & s_{33}^{E} & 0 & 0 & 0 & 0 & 0 & d_{33} \\
0 & 0 & 0 & s_{44}^{E} & 0 & 0 & 0 & d_{15} & 0 \\
0 & 0 & 0 & 0 & s_{44}^{E} & 0 & d_{15} & 0 & 0 \\
0 & 0 & 0 & 0 & 0 & 2\left(s_{11}^{E}-s_{12}^{E}\right) & 0 & 0 & 0 \\
0 & 0 & 0 & 0 & d_{15} & 0 & \varepsilon_{11}^{T} & 0 & 0 \\
0 & 0 & 0 & d_{15} & 0 & 0 & 0 & \varepsilon_{11}^{T} & 0 \\
d_{31} & d_{31} & d_{33} & 0 & 0 & 0 & 0 & 0 & \varepsilon_{33}^{T}
\end{array}\right) \times\left(\begin{array}{c}
T_{11} \\
T_{22} \\
T_{33} \\
T_{23} \\
T_{31} \\
T_{12} \\
E_{1} \\
E_{2} \\
E_{3}
\end{array}\right)
$$

where $s_{i j}^{E}$ is the elastic compliance at constant electric field, $\varepsilon_{k k}^{T}$ is the dielectric permittivity at constant stress and $d_{l m}$ is the piezoelectric constant. $S, T$ are the elastic field strain tensor and stress tensor, and $D, E$ are the electric displacement vector and field strength respectively. By convention, 1, 2 and 3 correspond to the $x$-, $y$ - and $z$-directions, respectively, and their corresponding normal planes.

Assuming that the drive electrode is in the polarization direction (direction 3), when an $\mathrm{AC}$ voltage $\left(V_{i n}=\right.$ $\left.V_{A} \sin \omega t\right)$ is applied to the drive electrode under the mechanical free boundary condition, the thickness extension vibration mode will be produced. There are three vibration strains in the thickness extension vibration mode. According to equation (2), the three strains are written as:

$$
\begin{aligned}
& S_{11}=d_{31} E_{3}=-\frac{V_{A} d_{31}}{c} \sin \omega t \\
& S_{22}=d_{31} E_{3}=-\frac{V_{A} d_{31}}{c} \sin \omega t \\
& S_{33}=d_{33} E_{3}=-\frac{V_{A} d_{33}}{c} \sin \omega t .
\end{aligned}
$$

For the vibration strain $S_{11}$, imagine the whole plate is divided into many slices along the $x$-axis, and each layer has the same length $\mathrm{d} x$. Then, the layer $(x, x+\mathrm{d} x)$ has the velocity:

$$
v_{1}=\frac{\mathrm{d}}{\mathrm{d} t}\left(S_{11} x\right)=-\frac{V_{A} d_{31} \omega \cos \omega t}{c} x
$$

where its direction is along the $x$-axis. Similar to equation (6), for vibration strains $S_{22}$ and $S_{33}$, their velocities are:

$$
\begin{aligned}
& v_{2}=\frac{\mathrm{d}}{\mathrm{d} t}\left(S_{22} y\right)=-\frac{V_{A} d_{31} \omega \cos \omega t}{c} y \\
& v_{3}=\frac{\mathrm{d}}{\mathrm{d} t}\left(S_{33} z\right)=-\frac{V_{A} d_{33} \omega \cos \omega t}{c} z
\end{aligned}
$$

where their directions are along the $y$-axis and $z$-axis, respectively.

The input rotation rate $\Omega$ is assumed to be about the $y$ axis. According to equation (1), only $S_{11}$ and $S_{33}$ vibration strains could produce a Coriolis force. Consider $S_{11}$ first. The layer $(x, x+\mathrm{d} x)$ will experience a Coriolis force:

$$
\mathrm{d} \vec{F}_{C}=2(\mathrm{~d} m) \vec{v}_{1} \times \vec{\Omega}=K\left(2 b d_{31} x \mathrm{~d} x\right) \hat{z}
$$




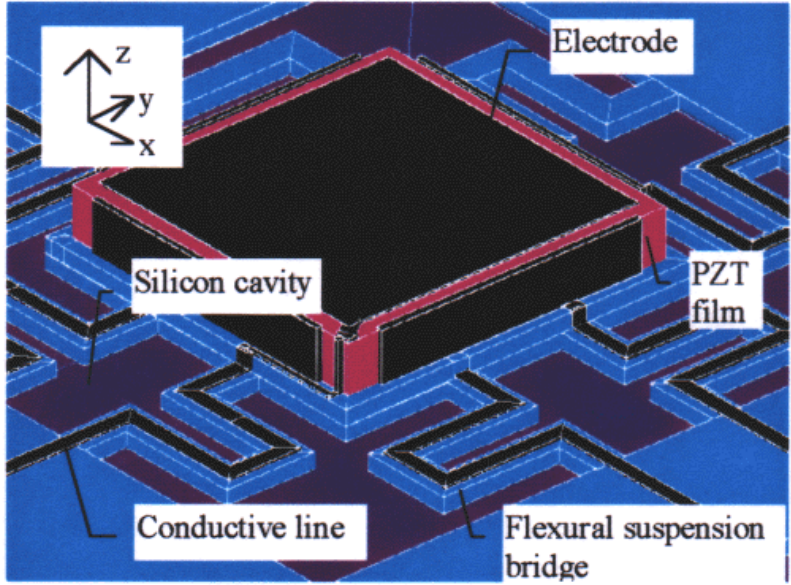

Figure 2. The piezoelectric plate two-axis microgyroscope.

where $K=-V_{A} \rho \omega \Omega \cos \omega t, \mathrm{~d} m$ is the mass of the layer $(x, x+\mathrm{d} x)$ and $\rho$ is the density of the piezoelectric plate. This Coriolis force $\mathrm{d} F_{C}$ is in the $z$-direction. Thus, the local Coriolis force applied to the layer $(x, x+\mathrm{d} x)$ can be treated as a shear stress $\left(\mathrm{d} T_{31}\right)$ which acts on the $y-z$-plane of the piezoelectric plate in the $z$-direction. For the whole piezoelectric plate, the shear stress will be:

$$
T_{31}=K d_{31} \frac{a^{2}}{4 c}
$$

According to equation (2), $T_{31}$ will produce a component of electric field in the $x$-direction and thus a voltage in the $x$ direction is induced. Denote this the output voltage signal $V_{O u t}^{\prime}$. Its amplitude is proportional to the input rotation rate $\left(K=-V_{A} \rho \omega \Omega \cos \omega t\right)$.

$$
V_{\text {Out }}^{\prime}=\frac{D_{1} a}{\varepsilon_{11}^{T}}=\frac{a d_{15} T_{31}}{\varepsilon_{11}^{T}}=\frac{K d_{31} d_{15}}{\varepsilon_{11}^{T}} \frac{a^{3}}{4 c} .
$$

Similar to equation (11), the $S_{33}$ strain will also produce an induced voltage $V_{O u t}^{\prime \prime}$ in the $x$-direction:

$$
V_{\text {Out }}^{\prime \prime}=-\frac{K d_{33} d_{15}}{\varepsilon_{11}^{T}} \frac{a c}{4} .
$$

Because $d_{31}$ is negative, $\left|d_{31}\right| \sim\left|d_{33}\right|$, and $a \gg c$ for a thin film piezoelectric microgyroscope structure, the total output voltage $V_{O u t}$ with thickness extension vibration mode is:

$$
\begin{aligned}
V_{\text {Out }} & =-\frac{K}{\varepsilon_{11}^{T}} \frac{a c}{4}\left(d_{33}+\left|d_{31}\right| \frac{a^{2}}{c^{2}}\right) d_{15} \\
\cong & \frac{V_{A} \rho \omega \cos \omega t}{\varepsilon_{11}^{T}} \frac{a^{3}}{4 c}\left|d_{31}\right| d_{15} \Omega .
\end{aligned}
$$

With the aforementioned analysis, the following conclusions can be reached:

(i) The piezoelectric plate gyroscope is well suited for scaling down to microdimensions. It has a potential advantage inherent in its piezoelectric film construction: the output voltage signal is proportional to $a^{3} / c$, where $a$ is the plate length and $c$ is the thickness. In these film devices, $c$ is of the order of $1 \mu \mathrm{m}$ and $a$ is of the order of $0.5-1 \mathrm{~mm}$. Therefore, the output voltage and the signalto-noise ratio will be large in the micro-piezoelectric plate gyroscope.

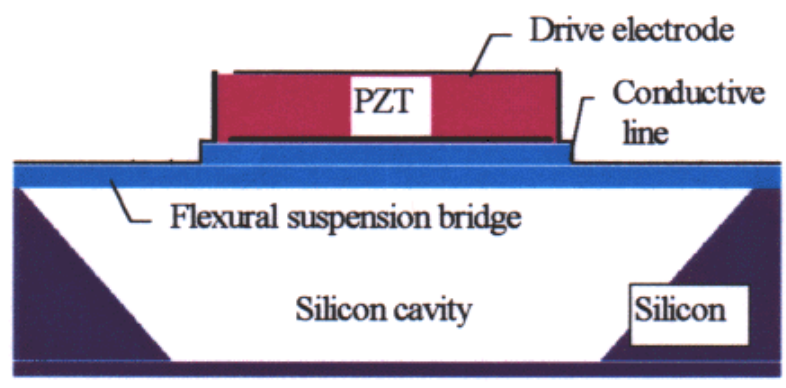

Figure 3. The cross section of the piezoelectric plate microgyroscope.

(ii) Using IC technology, the micro-piezoelectric gyroscope could be designed in cascade form because the output voltage signal is independent of the width in the $y$ direction. The output voltage will be amplified if several single-axis piezoelectric gyroscopes are cascaded.

(iii) It can be used as a dual-axis gyroscope if $a=b$ because the piezoelectric equation for PZT materials is symmetric in $x$ - and $y$-directions.

\section{An initial piezoelectric plate microgyroscope module}

A schematic diagram of the piezoelectric plate two-axis microgyroscope is shown in figure 2. It is based on silicon micromachining technology. The cross section of the structure is shown in figure 3. In order to achieve a stress-free piezoelectric plate which satisfies the mechanical free boundary condition, eight flexural suspension bridges are designed in the piezoelectric microgyroscope.

The PZT film is selected as the piezoelectric material not only because the piezoelectric constants of PZT is much larger than other piezoelectric films such as $\mathrm{ZnO}$ and $\mathrm{AlN}$, but also because the composition of PZT film can be accurately controlled and a $2 \mu \mathrm{m}$ thick PZT film is easy to achieve by the sol-gel fabrication processes [7]. In order to obtain the best performance (such as sensitivity, linearity, temperature property and fatigue characteristics) of the piezoelectric microgyroscope, some modifications of the piezoelectric material are required.

In our recent research, a layer of $2 \mu \mathrm{m}$ thick, crackfree PZT film on silicon has been achieved. It is shown that the PZT film has a completely perovskite structure and its XRD pattern (figure 4) is the same as PZT powder. The SEM micrograph of the PZT film also shows that its crystal structure is dense (figure 5). Therefore, in the following calculation for the piezoelectric plate microgyroscope, some material data of the PZT film are quoted from PZT crystals [8].

When the piezoelectric gyroscope is scaled down to microdimensions, the output voltage is very small, of the order of $0.1 \mu \mathrm{V}$. An on-chip lock-in amplifier is required in order to detect the small input angular rate. The fabrication process of PZT film by sol-gel technology is compatible with standard IC process. It is possible to integrate IC circuits together with the microgyroscope to fabricate an integrated 
Table 1. Specifications and performance of the initial piezoelectric plate two-axis microgyroscope.

\begin{tabular}{llll}
\hline \multicolumn{2}{c}{ Specifications } & \multicolumn{2}{c}{ Measuring conditions and performance } \\
\hline Piezoelectric material & $\mathrm{Pb}\left(\mathrm{Zr}_{0.54} \mathrm{Ti}_{0.46}\right) \mathrm{O}_{3}$ & AC drive voltage $V_{A}$ & $1 \mathrm{~V}$ \\
Density & $7.5 \times 10^{3} \mathrm{~kg} \mathrm{~m}^{-3}$ & Source frequency & $100 \mathrm{kHz}$ \\
Piezoelectric constant $d_{31}$ & $700 \times 10^{-12} \mathrm{~m} / \mathrm{V}$ & Lock-in amplifier $A_{K}$ & 10000 \\
Piezoelectric constant $d_{15}$ & $270 \times 10^{-12} \mathrm{~m} / \mathrm{V}$ & Thermal sensor noise & $<1 \times 10^{-3} \mathrm{~V}$ \\
Permittivity $\varepsilon_{11}^{T} / \varepsilon_{0}$ & 3250 & Maximum output voltage & $5 \mathrm{~V}$ \\
Plate length $a$ & $1000 \mu \mathrm{m}$ & Sensitivity $\left(A_{K} V_{\text {out }} / V_{A} / \Omega\right)$ & $0.0387 \mathrm{~V} / \mathrm{V} /(\mathrm{deg} / \mathrm{sec})$ \\
Plate width $b$ & $1000 \mu \mathrm{m}$ & Accuracy & $2.58 \times 10^{-2} \mathrm{deg} / \mathrm{sec}$ \\
Plate thickness $c$ & $2 \mu \mathrm{m}$ & Range & $129.2 \mathrm{deg} / \mathrm{sec}$ \\
\hline
\end{tabular}

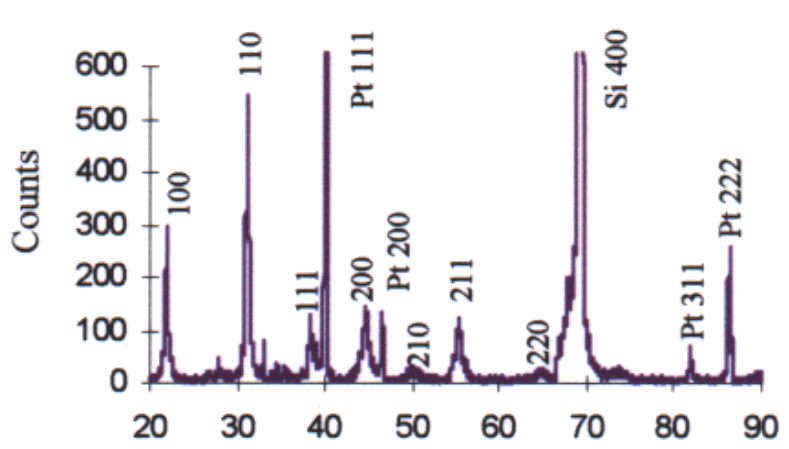

Degrees 2-Theta

Figure 4. XRD pattern of PZT film on $\mathrm{Pt} / \mathrm{Ti} / \mathrm{SiO}_{2} / \mathrm{Si}$.

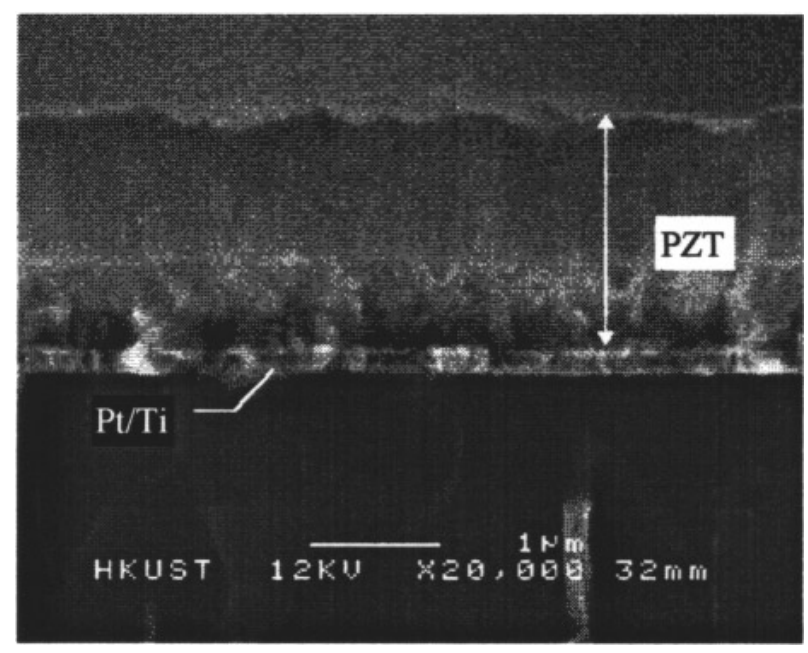

Figure 5. SEM micrograph of the PZT film.

MEMS. The integrated piezoelectric microgyroscope could have the sensitivity of $0.0387 \mathrm{~V} / \mathrm{V}(\mathrm{deg} / \mathrm{sec})$, accuracy of $0.026 \mathrm{deg} / \mathrm{sec}$ and measurement range of $129 \mathrm{deg} / \mathrm{sec}$ if the maximum output voltage of the on-chip IC is $5 \mathrm{~V}$ (which is estimated in table 1).

The resonant frequency of the thickness extension vibration mode of the piezoelectric plate depends on the piezoelectric material, the thickness of the plate and the supporting bridges material and their size [9]. A layer of $\mathrm{SiO}_{2}$ film or $\mathrm{SiO}_{2}$ and $\mathrm{Si}_{3} \mathrm{~N}_{4}$ composite film provides a good supporting material if silicon micromachining technology is used to fabricate the microgyroscope. The analysis above

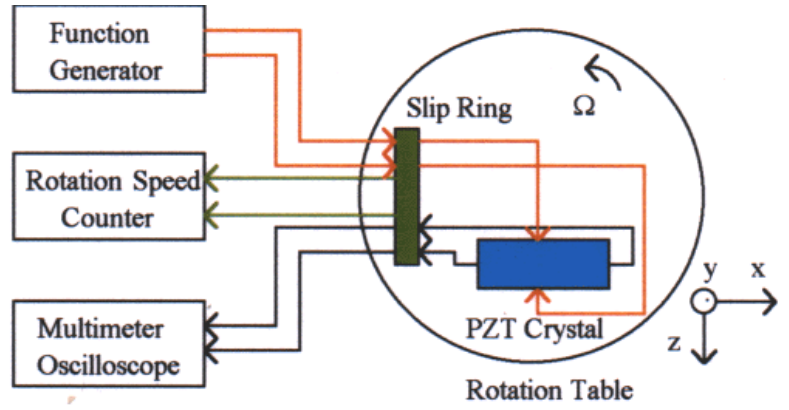

Figure 6. Experimental set-up for the piezoelectric plate gyroscope of PZT crystal.

yields an example design given in table 1, together with the performance predictions.

\section{A macro-model simulation experiment}

While the fabrication of the piezoelectric plate microgyroscope is currently under way, it is necessary to verify its fundamental theory by experiment using a commercially available macro-piezoelectric crystal. A PZT crystal bought from American Piezo Ceramics has dimensions of $20 \mathrm{~mm} \times 6 \mathrm{~mm} \times 5 \mathrm{~mm}$. It is constructed with conductiveepoxy electrodes screen-coated on six surfaces. There are small gaps between the conductive electrodes at the edges to avoid electrical short circuit. The basic experimental set-up is shown in figure 6.

In order to measure the output voltage of the PZT crystal gyroscope versus the input rotation rate, a function generator, multimeter, oscilloscope, rotation speed counter and rotation table are required in the experiment. The PZT crystal is glued on the rotation table by two-sided adhesive tape. The drive electrodes are located on the $z$-surfaces, and the signal electrodes on the $x$-surfaces. Leads attached to the drive and signal electrodes take the form of thin copper wires and are in spring-loaded contact with the slip ring. The slip ring thus provides the means by which the drive sends AC power to the rotating crystal, and by which the output signal is delivered to the multimeter.

The experimental procedure is as follows. First, the resonant frequency of the thickness extension vibration mode in the $z$-axis of the PZT crystal was determined by a direct frequency sweep using a function generator and oscilloscope 
Table 2. All possible configurations of piezoelectric plate gyroscope and the corresponding sensitivity.

\begin{tabular}{llllll}
\hline & $\begin{array}{l}\text { Direction of } \\
\text { Crive/input/sense }\end{array}$ & $\begin{array}{l}\text { Output sensitivity } \\
\frac{V_{O u t}}{V_{A} \cos \omega t \Omega}\end{array}$ & $\begin{array}{l}\text { Theory sensitivity } \\
(\mu \mathrm{V} / \mathrm{V}(\mathrm{deg} / \mathrm{sec}))^{\mathrm{a}}\end{array}$ & $\begin{array}{l}\text { Resonant } \\
\text { frequency }(\mathrm{kHz})\end{array}$ & $\begin{array}{l}\text { Experimental } \\
\text { sensitivity } \\
(\mu \mathrm{V} / \mathrm{V}(\mathrm{deg} / \mathrm{sec}))\end{array}$ \\
\hline $\mathrm{A}$ & $x / y / z$ & $\left(\rho \omega / \varepsilon^{T_{33}}\right)\left(d_{33} d_{15}\right)\left(c^{3} / 4 a\right)$ & 0.34 & 180 & 0.06 \\
$\mathrm{~B}$ & $x / z / y$ & $\left(\rho \omega / \varepsilon^{T_{11}}\right)\left(d^{215}\right)\left(b c^{2} / 4 a\right)$ & 0.13 & 183 & 5.35 \\
$\mathrm{C}^{\mathrm{b}}$ & $y / x / z$ & $\left(\rho \omega / \varepsilon^{T_{33}}\right)\left(\left|d_{31}\right| d_{15}\right)(b c / 4)$ & 0.11 & 200 & 0.06 \\
$\mathrm{D}$ & $y / z / x$ & $\left(\rho \omega / \varepsilon^{T_{11}}\right)\left(d^{25}\right)\left(a c^{2} / 4 b\right)$ & 4.85 & 205 & 18.28 \\
$\mathrm{E}$ & $z / x / y$ & $\left(\rho \omega / \varepsilon^{T_{11}}\right)\left(\left|d_{31}\right| d_{15}\right)\left(b^{3} / 4 c\right)$ & 0.51 & 216.5 & 1.68 \\
$\mathrm{~F}^{\mathrm{c}}$ & $z / y / x$ & $\left(\rho \omega / \varepsilon^{T_{11}}\right)\left(\left|d_{31}\right| d_{15}\right)\left(a^{3} / 4 c\right)$ & 37.56 & 214.5 & 34.76 \\
\hline
\end{tabular}

${ }^{a}$ Some data used in calculation are quoted from [8].

b Hansjoachim's design (macrogyroscope) [1].

c Our design (microgyroscope).

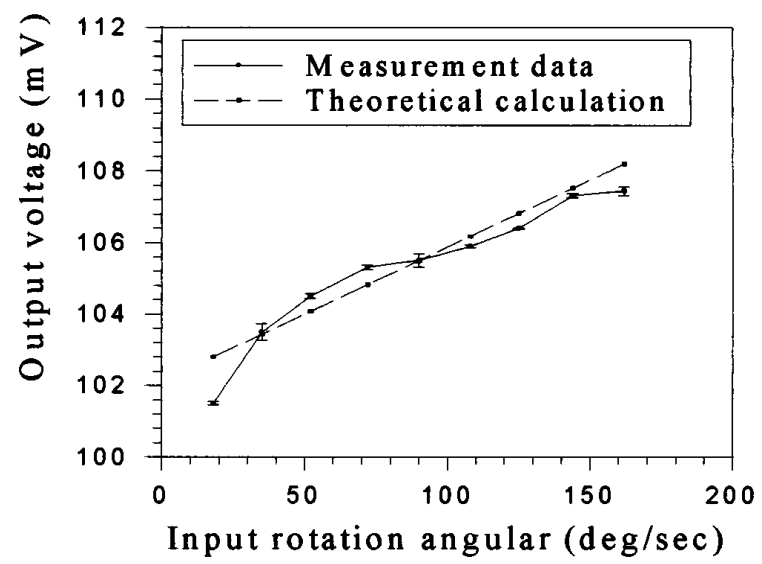

Figure 7. Output voltage of PZT crystal piezoelectric gyroscope versus input rotation rate.

when the input rotation rate was zero. Second, the function generator was set to sine wave output with an amplitude of 1 volt and a frequency equal to the thickness extension vibration resonant frequency. Then, the relationship of the output voltage signal in the $x$-direction to the input angular velocity was measured by a multimeter and a rotation speed counter. Figure 7 shows the measured results. The theoretical predictions are also presented in figure 7 while some data used in calculation are quoted from [8]. It can be seen that the experimental results are very close to the theoretical calculation if an offset is added to the theory.

\section{Concluding remarks}

In this paper, a piezoelectric plate microgyroscope has been proposed. Its principle has been verified by theory and experiments. It merits attention especially in MEMS systems because its structure is suitable for IC fabrication and thus it is easy to integrate signal processing circuits on the same chip. The results of the present study can be summarized as follows.

(a) According to the physical model given in equation (13), the output voltage of the piezoelectric plate gyroscope will be zero in the absence of an input rotation rate. However, a $100 \mathrm{mV} \mathrm{AC}$ output voltage is present in the experiments even if the rotation table is not rotating. This output voltage is fed through produced by the drive signal, and is very difficult to eliminate. The theoretically calculated straight line is close to the experimental results if the average value of the measurement data is used as an offset. The slope of this line is the theoretical sensitivity, and is $37.56 \mu \mathrm{V} / \mathrm{V}(\mathrm{deg} / \mathrm{sec})$. The sensitivity of experimental measurement is estimated to be about $34.76 \mu \mathrm{V} / \mathrm{V}(\mathrm{deg} / \mathrm{sec})$ by a least squares fit, and matches theoretical prediction closely. The small difference between experiment and theory may be due to experimental error because the PZT crystal is glued on the rotating table by adhesive tape and the complete mechanical free boundary condition of the piezoelectric plate gyroscope physical model is not exactly satisfied.

(b) For a piezoelectric plate, if it is driven in the $x$ - or $y$ direction rather than in the $z$-direction, other vibration modes will be produced. All vibration modes can be used to detect the input rotation rate. Hamisch Hansjoachim [1] used the thickness shear vibration mode to measure angular rate by driving the PZT piezoelectric plate in the $y$-direction and detecting the output signal voltage in the $z$-direction. Table 2 lists all possible driving and detection configurations of the piezoelectric plate gyroscope and their output sensitivities. The theoretical calculations and experimental results are based on the PZT crystal which is described before. It is shown that the output sensitivity of the piezoelectric plate gyroscope with thin film structure $(a \gg c, b \gg c)$ predicted in this paper is the largest, and the experimental results are close to the theoretical calculations.

\section{References}

[1] Hansjoachim $\mathrm{H}$ et al 1990 Sensor for determining angular velocity World Patent WO 90/07124

[2] Söderkvist J 1994 Micromachined gyroscopes Sensors Actuators A 43 65-71

[3] Greiff P et al 1991 Silicon monolithic micromechanical gyroscope Transducers'91 (San Francisco, 1991) pp 966-8

[4] Tanaka K et al 1995 A micromachined vibrating gyroscope Sensors Actuators A 50 111-5 
[5] Bernstein J et al 1993 A micromachined comb-drive tuning fork rate gyroscope Proc. Micro Electro Mechanical Systems (Fort Lauderdale, 1993) pp 143-8

[6] Shun Kang and Zhang Fuxue 1984 Piezoelectricity (Beijing: Defence Industry Press) p 134

[7] Yi Guanghua et al 1992 Sol gel processing of thick PZT films ISAF'92, Proc. 8th IEEE Int. Symp. on Applications of Ferroelectrics (1992) pp 289-92
[8] Landolt-Börnstein New Series Group III, vol 16a (Berlin: Springer) $\mathrm{p} 123$

[9] Muralt P et al 1996 Piezoelectric actuation of PZT thin-film diaphragms at static and resonant conditions Sensors Actuators A $\mathbf{5 3} 398-404$ 